$<1$ group (Group HFNEF, 20 cases). In addition, Group HFREF (LVEF $<45 \%, 62$ cases) was divided into 3 parts on the basis of New York Heart Association (NYHA) classification of the cardiac function: Grade II group (Group HF1, 20 cases), Grade III group (Group HF2, 21 cases) and Grade IV group (Group HF3, 21 cases). ELISA method was used to detect ACT-A and BNP levels in serum of patients with CHF and the control group in our study.

Results Serum ACT-A was in a state of high expression in patients with heart failure compared with the control group. The levels of serum ACT-A in Group HFREF, Group HFNEF and the normal control group (Group $\mathrm{N}$ ) were (1.81 \pm 0.44$)$, (1.36 \pm 0.28$)$, $(1.24 \pm 0.18) \mathrm{ng} / \mathrm{ml}$, and the difference between every two groups was significant $(p<0.01-0.05)$. The expression levels of serum ACT$A$ in patients of Group HFREF was positively correlated with the degree of heart failure $(r=0.75)$. Serum ACT-A and BNP in patients with heart failure presented a positive correlation $(r=0.82)$.

Conclusions ACT-A is expected to be regarded as the effective clinical serology index, and has an important reference value to the diagnosis of heart declines.

\section{e0613 PLASMA MICRORNA-361-5P AS A BIOMARKER OF CHRONIC HEART FAILURE}

doi:10.1136/hrt.2010.208967.613

Chen Chen, Yang Shenglan, Wang Feng, Long Guangwen, Yang Xu, Chen Fuqiong, Wang Dao Wen. Tongji

Aims Recent studies have showed important roles for microRNAs in multiple cardiovascular diseases, including AMI, hypertension, HF, and so on. However, the signature of plasma miRNA expression in chronic heart failure has not been well understood.

Methods Here we used array analysis of miRNA production in plasma of heart failure patients $(n=10)$ and normal control people $(n=10)$ to identify the heart failure-specific miRNAs. And the results were confirmed by miRNA real-time PCR assay. Then, we assessed the plasma concentrations of miRNA in 20 individuals with acute coronary syndromes using a miRNA real-time PCR method that use U6 as an internal reference.

Results The miRNA microarray analysis of human plasma from heart failure patients and normal control people indicated that plasma miR-361-5p concentrations were decreased significantly in all individuals with heart failure. However, in contrast with the expression in heart failure, plasma miR-361-5p concentrations were markedly increased in all individuals with AMI.

Conclusions Our data demonstrated that the plasma concentrations of miR-361-5p were obviously various in heart failure patients, AMI patients and normal control people. Thus, plasma miR-361-5p may reveal a new biomarker for chronic heart failure.

\section{e0614 LV DIASTOLIC RELAXATION AND FILLING ASSESSMENT USING THE TIME INTERVAL BETWEEN MITRAL INFLOW AND MITRAL ANNULAR VELOCITIES}

doi:10.1136/hrt.2010.208967.614

${ }^{1}$ Huang Feiqiong, ${ }^{2}$ Zhang Ruisheng, ${ }^{1}$ Le Thuthao, ${ }^{1}$ Tan Rusan. ${ }^{1}$ National Heart Centre Singapore, Singapore, ${ }^{2}$ Beijing Hospital, Beijing, China

Objective The aim of ours study was to evaluate the left ventricular (LV) function using the time intervals between peak mitral inflow and peak mitral annular velocities.

Methods This study included 39 patients with heart failure, left ventricular hypertrophy $(\mathrm{LVH})$ and ischaemic heart disease (age from 29 to 56 years old, mean age: 36 years), and 23 age-matched healthy controls (age from 30 to 50 years). According to the filling pattern, patients were classified into 2 groups: (1) impaired relaxation group and (2) restrictive filling pattern group. The measurements were: the time intervals from the R-wave on the ECG to the peak E-wave on the transmitral flow (TMF) (R-pE), to the peak E'wave on the LV lateral wall of tissue Doppler imaging (TDI) (R-pE'); The time intervals from the onset of P-wave on the ECG to the peak A-wave on the TMF (P-pA), to the peak $A^{\prime}$-wave (P-pA') on TDI. Early-diastolic temporal discordance (EDTD) and late-diastolic temporal discordance (LDTD) were calculated as the difference between the time intervals (R-E) and (R-E'), (P-pA) and (P-p A').

Results EDTD and LDTD were significantly decreased in the impaired relaxation group and restrictive filling pattern group compared with control group respectively $(20.6 \pm 28.3,9.1 \pm 14.3 \mathrm{vs}$ $34.5 \pm 22.3, \quad \mathrm{p}<0.001), \quad(16.4 \pm 15.2, \quad 3.5 \pm 22.6$ vs $31.4 \pm 13.0$, $\mathrm{p}<0.001)$

Conclusions EDTD and LDTD, which mean the LV relaxation and left atrial contraction, may be a useful new method to evaluate the LV diastolic function in patients with heart diseases.

\section{e0615 THE RELATIONSHIP BETWEEN LEFT VENTRICULAR DIASTOLIC FUNCTION AND ARTERIAL STIFFNESS IN CORONARY HEART DISEASE PATIENTS WITH DIABETES}

doi:10.1136/hrt.2010.208967.615

Yankai Lian, Yongliang Wang, Hongwei Li, Yongquan Wu. Friendship Hospital, Washington, District of Columbia, USA

Objectives By measuring left ventricular diastolic function and arterial stiffness, this study aims to probe into the effect of diabetes mellitus (DM) on left ventricular diastolic function and arterial stiffness, and evaluate the correlation between left ventricular diastolic function and arterial stiffness.

Materials and methods 76 inpatients who performed both coronary angiography and left ventricular angiography at the cardiovascular center of Beijing Friendship Hospital Affiliated with Capital Medical University from August 2009 to February 2010 were enrolled. Those with no diabetic history were given oral glucose tolerance test (OGTT). According to their coronary angiography, OGTT test results and past history of DM, patients were divided into controlled, CHD (Coronary Heart Disease) (with no DM), and CHD + DM groups. Through invasive haemodynamic monitoring during left ventricular angiography, left ventricular end-diastolic pressure (LVEDP) and tau index were collected. Carotid-femoral pulse wave velocity (c-f PWV), reflected wave augmentation index (AIx@75) and other data reflecting the degree of arterial stiffness were collected bedside with non-invasive means. SPSS 18.0 was used for statistical analysis

Results (1) $37.5 \%$ (21 cases) of CHD patients were previous diagnosed type 2 diabetes before admission. Of those with no previous history of DM, 20\% (7 cases) had impaired glucose regulation, and $28.6 \%$ (10 cases) were newly diagnosed type 2 diabetes. Overall, 67.9\% (38 cases) of CHD patients also had abnormal glucose metabolism. (2) No significant differences were found between groups for left ventricular end diastolic pressure (LVEDP), tau index, and AIx@75. In terms of c-f PMV. The CHD + DM group $(8.79 \pm 1.59 \mathrm{~cm} / \mathrm{s})$ differ significantly from the CHD group $(7.43 \pm 1.42 \mathrm{~cm} / \mathrm{s})$ and the controlled group $(6.83 \pm 1.14 \mathrm{~cm} /$ $\mathrm{s})$. No correlations were found between c-f PMV and LVEDP or tau index. A positive correlation was found between AIx@75 and tau index.

Conclusions (1) Compared to the controlled group and $\mathrm{CHD}$ patients with no DM, CHD+DM patients showed worse arterial stiffness. No differences were found for left ventricular diastolic function. (2) There is a positive correlation between arterial stiffness and diastolic dysfunction. 